\title{
Left ventricular noncompaction associated with hypertrophic cardiomyopathy and Wolff-Parkinson-White syndrome
}

\author{
Luis Alday ${ }^{1}$, Eduardo Moreyra $^{1}$, Eva Bruno $^{2}$, Norma Rossi $^{3}$, Hector Maisuls $^{2}$ \\ ${ }^{1}$ Divisions of Pediatric Cardiology and Cardiology, Sanatorio Allende, Córdoba, Argentina; lealday@arnet.com.ar \\ ${ }^{2}$ Division of Cardiology, Children's Hospital, Cordoba, Argentina \\ ${ }^{3}$ Division of Genetics, Private Hospital, Cordoba, Argentina
}

Received 14 October 2009; revised 10 December 2009; accepted 14 December 2009.

\begin{abstract}
We report a 35-year-old female patient with hypertrophic cardiomyopathy, left ventricular noncompaction, and Wolff-Parkinson-White EKG pattern. Several other family members present the same clinical condition. We speculate that this phenotype is related to the genotypes PRKAG2 and LAMP2 represented by mutations of the genes encoding AMP-activated protein kinase (PRKAG2) and lysosome associated membrane protein 2 (LAMP2).
\end{abstract}

Keywords: Left Ventricular Noncompaction; Hypertrophic Cardiomyopathy; Wolff-Parkinson-White Syndrome

\section{INTRODUCTION}

Left ventricular noncompaction (LVNC) is the result from the arrest in myocardial development with persistent sinusoid tracts interspersed with prominent muscular trabeculae. Affected patients usually develop congestive heart failure associated with arrhythmias and systemic thromboembolism.We report a proband with mental retardation and peculiar somatic findings, associated LVNC and hypertrophic cardiomyopathy (HCM). Other family members were also involved, all of them, the proband included, had Wolff-Parkinson-White (WPW) syndrome, suggesting a relationship regarding the etiology of this LVNC - HCM phenotype overlapping.

\section{CASE REPORT}

The proband is a 35-year-old female who had been followed since infancy. She was referred to us for a heart murmur and congestive heart failure. Clinical examination at presentation was compatible with moderate to severe mitral regurgitation. There was cardiomegaly and signs of pulmonary venous hypertension on the chest X-ray. The EKG showed a WPW pattern with extremely high voltage. Cineangiography revealed a hypercontractile left ventricle without outflow tract obstruction, moderate to severe mitral regurgitation, and a never-seen-before contour which made it resemble a "porcupine" since there were heavy trabeculations leaving thin spaces between them as if they were wheel spokes radiating from the center to the periphery of the ventricular cavity. On the basis of the angiographic findings this patient's heart was called a "spongy" heart because was thought to be the result of persistence of embryonic myocardium [1].

The patient was treated for heart failure until early childhood. There was clinical improvement with decreasing mitral regurgitation as judged by auscultation. She remained symptom free for several years and she had non-obstructive HCM with mild mitral insufficiency upon echocardiographic examination. She continued regular follow-up visits and the echocardiograms showed progression to a dilated stage. Eight years ago she was admitted with pulmonary edema and frequent ventricular extrasystoles. Following treatment she was in NYHA functional class II and was discharged. Occasional remissions occurred caused by treatment non-compliance.

Presently, she is in functional class III and has slight mental retardation. She has lower than normal stature, a short neck, low posterior hairline, and a high palate. Clinical genetics evaluation suggests the diagnosis of Noonan syndrome. Cardiovascular examination shows, a mitral regurgitation murmur and gallop rhythm. Chest $\mathrm{x}$-ray and EKG presented severe cardiomegaly with signs of pulmonary venous hypertension and WPW pattern, respectively. Color Doppler-echocardiography demonstrated hypertrophic and dilated left ventricle with prominent cavitary trabeculae compatible with the diagnosis of LVNC (LVNC: C ratio > 2.0), poor contractility, moderate mitral regurgitation, and pericardial effusion (Figure 1(a)). 


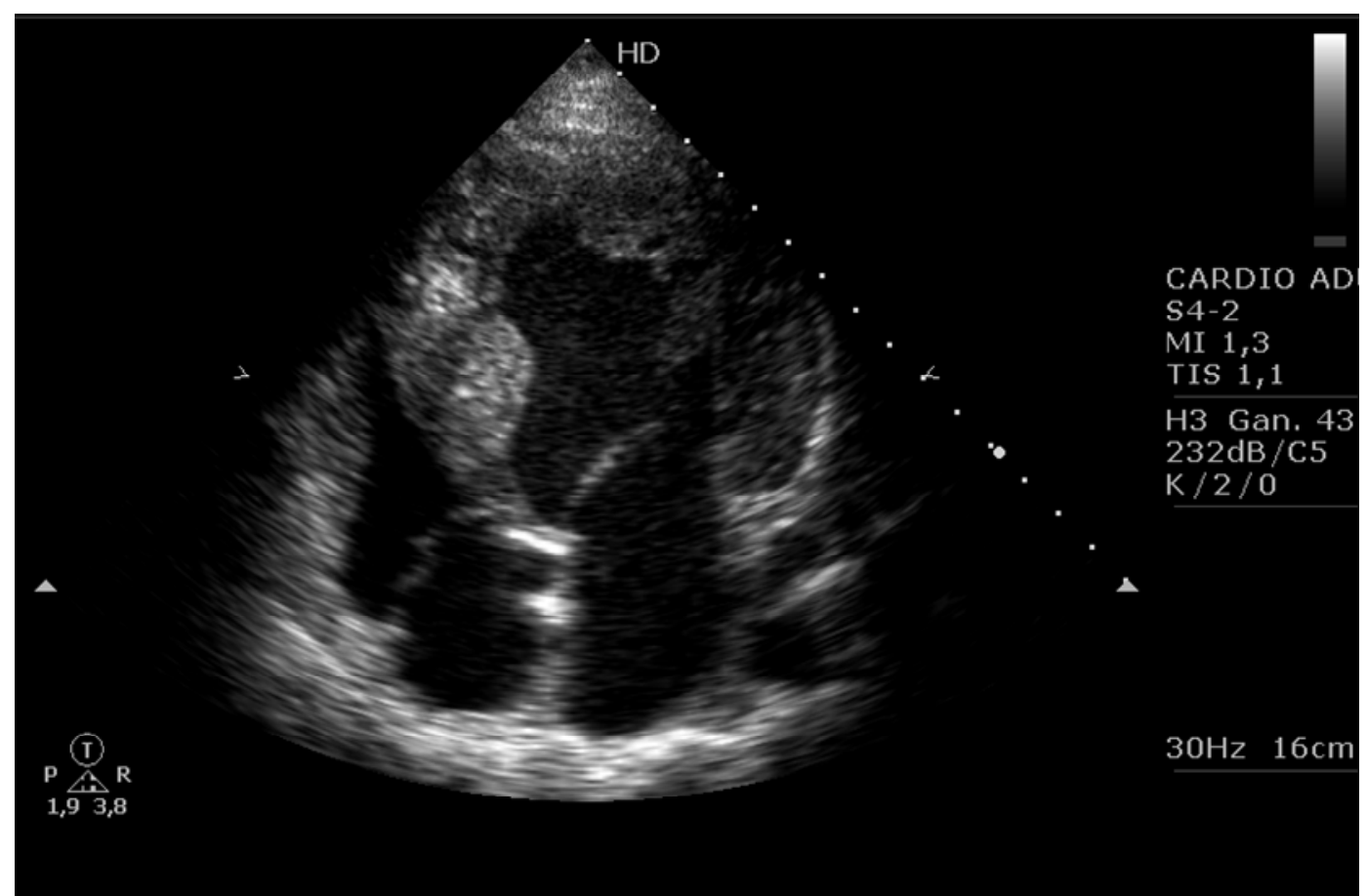

(a)

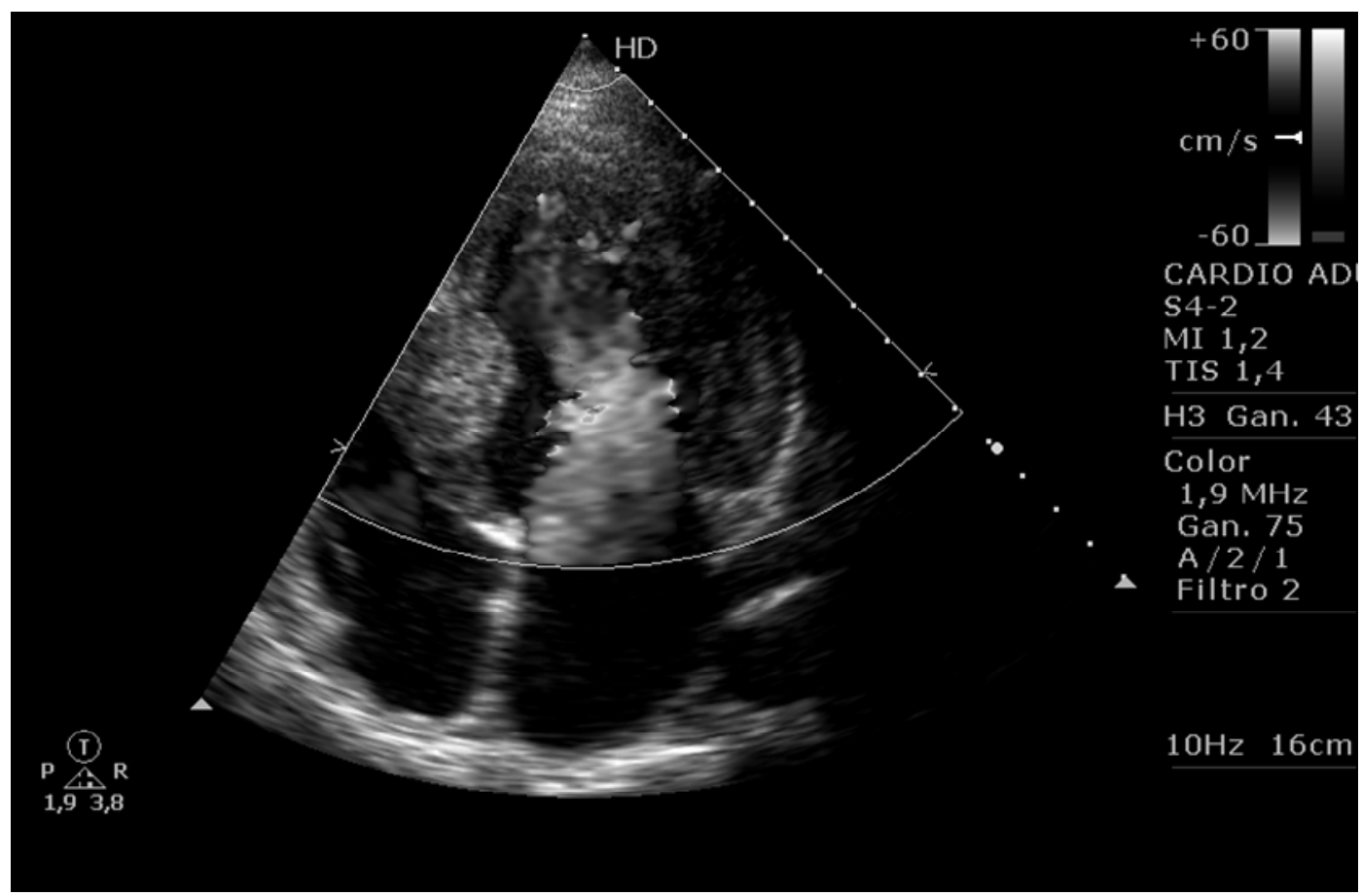

(b)

Figure 1. (a) Echocardiographic four-chamber view of the proband; There is hypertrophic cardiomyopathy (HCM) and left ventricular noncompaction (LVNC) with heavy trabeculations in the left ventricular (LV) cavity. The interventricular septum (IVS): LV posterior wall (PW) ratio is 1.4:1.0. The noncompacted (NC): compacted (C) ratio is $>2.0$. The LV end diastolic (ED) diameter is 6.0 and the ejection fraction (EF) 23\%. Pericardial effusion is present; (b) Color-Doppler echocardiographic four-chamber view of the proband's sister. There is also HCM and LVNC. The color in the LV cavity delineates the heavy trabeculae. The NC: C ratio $>2.0$. There is concentric LV hypertrophy with a prominent septal bulge. The IVS: PW ratio is 1.8:1.0. The LVED diameter and the EF are $4.9 \mathrm{~mm}$ and $56 \%$ respectively. 

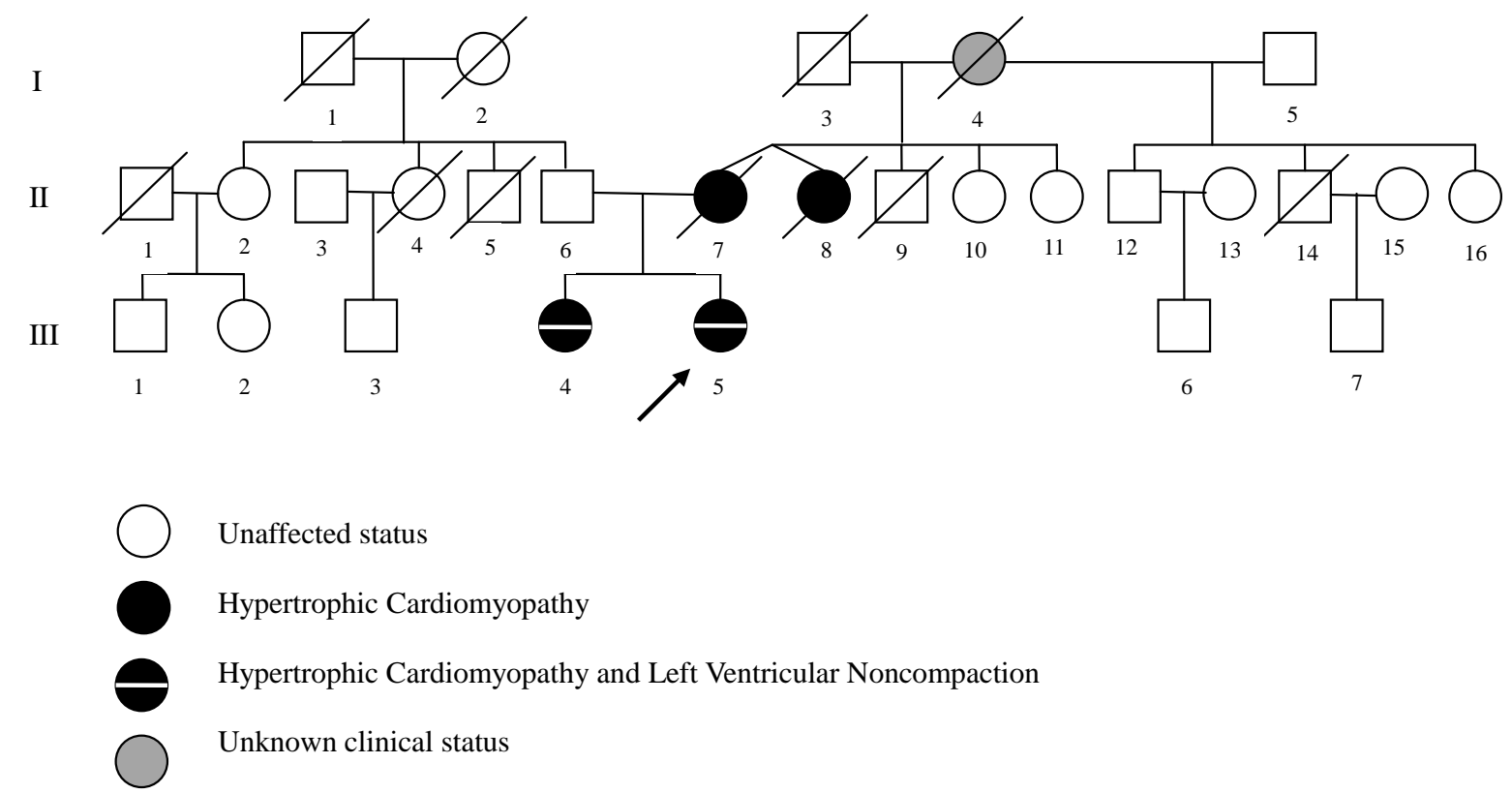

Figure 2. Pedigree of the kindred with hypertrophic cardiomyopathy (HCM), Wolff-Parkinson-White syndrome (WPW), and left ventricular noncompaction (LVNC) inherited as a Mendelian autosomal dominant trait.The proband is pointed by the arrow. I4: Proband's grandmother with unknown clinical status. II7 and 8: Proband's mother and aunt both of them with HCM and WPW. III4: Proband's sister with HCM, WPW, and LVNC.

The better resolution of the new echocardiographic technology allowed confirmation of the initial cineangiography. An updated family tree (Figure 2) showed an interesting family pattern where the proposita had a 37-year-old sister also with non-obstructive HCM with non-compaction and normal left ventricular function (Figure 1(b)). External features and mental status were normal. She also had a WPW pattern on the EKG with normal voltage. She had occasional episodes of supraventricular tachycardia. Both the mother and her identical twin had HCM, WPW, and atrial fibrillation. They might have had also LVNC but could not be confirmed. They died one year apart at the ages of 59 and 60 years due to congestive heart failure and stroke, respectively. The mother's sister had no descendants. The twins had 3 siblings and half- siblings each with no history of HCM. The maternal grandmother died suddenly at a young age of unspecified causes.

\section{DISCUSSION}

LVNC was a poorly recognized entity that began to gain interest from the medical community in the 90's. Recently, it has become clear that is more prevalent than previously recognized [2]. However, its etiology remained unknown until recently, when molecular genetic studies showed that mutations of cytoskeletal genes like those encoding taffazin (TAZ) and alpha-dystrobrevin (DTNA) causing Barth syndrome were associated with LVNC [3]. More recently, it was found that LVNC was also associated with mutations of sarcomeric genes shared by patients with HCM and dilated cardiomyopathy, like the alpha-cardiac actin gene (ACTC), beta-myosin heavy chain (MYH7), and cardiac troponin T (TNNT2) [4,5]. Cases of patients sharing both the LVNC and HCM phenotypes have been published recently but there was no mention of a mutation $[6,7]$.

The patient reported here, had other family members with the same disease, and all had the associated WPW syndrome. Such overlapping phenotype suggests a strong genetic association.

WPW syndrome is infrequently associated with HCM. It has been found in patients with HCM phenotypes with mutations of the genes encoding AMP-activated protein kinase (PRKAG2) and lysosome associated membrane protein 2 (LAMP2). PRKAG2 is an important enzyme involved in the production of cellular energy and its mutations might cause HCM, WPW syndrome, conduction disorders requiring pacing, skeletal myopathy, and glycogen storage disease [8]. About 30\% of affected individuals have atrial fibrillation or supraventricular tachycardia. This clinical pattern was seen in three of the family members of our proposita.

LAMP-2, an X-linked gene, encodes proteins that regulate the integrity and function of lysosomes. Mutations of this gene cause Danon disease characterized by HCM, WPW syndrome, skeletal myopathy and mental retardation. Female carriers present later in life with less severe disease than in males [9]. 
In the family under discussion, the proband and the sister had a phenotype of HCM, WPW syndrome, and LVNC. The proband had also somatic features suggestive of Noonan syndrome and mental retardation. The diseased mother and her twin, with normal external phenotype, had had HCM, WPW syndrome, and late onset atrial fibrillation, but LVNC could not be confirmed.

The members of this family had features shared with patients affected with the PRKG2 and LAMP-2 mutations. It is then possible that any of the two might be the involved gene. There are no systematic molecular genetic studies available showing the association of these genes with the HCM, WPW and LVNC phenotypes.

The complex overlapping phenotypes of HCM with WPW syndrome and LVNC seen in this family is possibly due to a previously not described mutation which should be studied further.

\section{REFERENCES}

[1] Van Praagh, R., Personal communication.

[2] Pignatelli, R.H., McMahon, C.J., Dreyer, W.J., et al. (2003) Clinical characterization of left ventricular noncompaction in children: a relatively common form of cardiomyopathy. Circulation, 108, 2672-2678.
[3] Ichida, F., Tsubata, S., Bowles, K.R., et al. (2001) Novel gene mutations in patients with left ventricular noncompaction or Barth syndrome. Circulation, 103, 1256-1263.

[4] Monserrat, L., Hermida-Prieto, M., Fernandez, X., et al. (2007) Mutation in the alpha-cardiac actin gene associated with apical hypertrophic cardiomyopathy, left ventricular non-compaction, and septal defects. European Heart Journal, 28, 1953-1961.

[5] Klaassen, S., Probst, S., Oechslin, E., et al. (2008) Mutation in sarcomere protein genes in left ventricular noncompaction. Circulation, 117, 2893-2901.

[6] Pantazis, A.A., Kohll, S.K., and Elliott, P.M. (2006) Images in cardiology. Hypertrophic cardiomyopathy and left ventricular hypertrabeculation: evidence for an overlapping phenotype. Heart, 92, 349.

[7] Kelley-Hedgepeth, A., Towbin, J.A., and Maron, M.S. (2009) Images in cardiovascular medicine. Overlapping phenotypes. Left ventricular noncompaction and hypertrophic cardiomyopathy. Circulation, 119, 588-589.

[8] Murphy, R.T., Mogensen, J., McGarry, K., et al. (2005) Adenosine monophosphate-activated protein kinase disease mimicks hypertrophic cardiomyopathy and WolffParkinson-White syndrome. Natural history. Journal of the American College of Cardiology, 45, 922-930.

[9] Yang, Z., McMahon, C.J., and Smith, L.R. (2005) Danon disease as an underrecognized cause of hypertrophic cardiomyopathy in children. Circulation, 112, 1612-1617. 